\title{
Year in Review 2014: COPD
}

\author{
Ruben D Restrepo MD RRT FAARC
}

\author{
Introduction \\ Disease Management Programs \\ Exercise Training \\ Smoking, Depression, and Anxiety in COPD \\ Respiratory Therapist-Driven Protocols and COPD \\ Pharmacotherapy \\ Summary
}

\begin{abstract}
Clinicians responsible for treating pulmonary disease often encounter challenges in the management of patients with COPD. This is due in part to the number of drugs now available to ameliorate COPD symptoms and the complexity of adhering to good disease management programs. Each aspect of treatment is a critical component in improving outcomes for these patients. The purpose of this article is to review some of the most significant findings regarding the treatment of COPD, with emphasis on disease management and pharmacotherapy. Key words: disease management; pharmacotherapy; pulmonary rehabilitation; exercise training; inhaled therapy; COPD exacerbation; respiratory therapist-driven protocols. [Respir Care 2015;60(7):1057-1060. () 2015 Daedalus Enterprises]
\end{abstract}

\section{Introduction}

Significant progress has been made in understanding the pathophysiology of COPD. However, management of COPD continues to be challenging to the clinician. An explosion of research in all aspects of COPD has not been the exception in 2014. A search of the PubMed database with the term COPD for 2014 and limited to the English

Dr Restrepo is affiliated with The University of Texas Health Science Center at San Antonio, San Antonio, Texan.

Dr Restrepo presented a version of this paper at the Year in Review 2014 of the AARC Congress 2014, held December 9-12, 2014, in Las Vegas, Nevada.

Dr Restrepo discloses relationships with Covidien, Fisher \& Paykel Healthcare, Hill-Rom, and Teleflex Medical.

Correspondence: Ruben D Restrepo MD RRT FAARC, Department of Respiratory Care, The University of Texas Health Science Center at San Antonio, MSC6248, San Antonio, TX 78229. E-mail: restrepor@uthscsa.edu.

DOI: $10.4187 /$ respcare. 04227 language and human trials resulted in 691 references. Only $4 \%(150 / 4,039)$ of the manuscripts came from clinical trials dealing with the treatment of stable COPD. This summary is based on 16 selected clinical trials and systematic reviews that evaluated 2 important aspects in the treatment of COPD: disease management and pharmacotherapy. This summary will emphasize the areas of pulmonary rehabilitation, exercise training, and impact of depression and anxiety. With regard to pharmacotherapy, a selection of papers on specific agents and the role of medication and inhalation technique are discussed.

\section{Disease Management Programs}

The primary goal of a disease management program for patients with COPD is to improve health outcomes and reduce health-related costs. These programs can be institution-, community-, or home-based. They also can vary in prescriptions of exercise and the level of follow-up by health-care providers. Mehring et $\mathrm{al}^{1}$ evaluated a disease management program for COPD in a longitudinal population-based study in Bavaria, Germany, and compared the total disease management program population of up to 
86,560 subjects with a stable cohort of 17,549 subjects over a period of $5 \mathrm{y}$. They reported significant declines of 1 and $2 \% / y$ in the use of systemic corticosteroids and theophylline, respectively $(P<.001)$. The occurrence of COPD exacerbations decreased at a rate of $0.9 \%$ in the total population. The proportion of smokers decreased by $8 \%$ even after dropout adjustment.

Lou et $\mathrm{al}^{2}$ reported the efficacy of a complex community-based COPD disease management program in 8,217 subjects with COPD between May 2008 and May 2012. The disease management program included assessment of the subjects' health status, delivery of regular health lectures, smoking cessation counseling, encouragement of regular exercise, rehabilitative training and psychological counseling, and regular follow-up. They found that the mean cumulative value of the BODE (body mass index, air-flow obstruction, dyspnea, exercise capacity) index increased by $0.7 /$ subject in the control group and by $0.4 /$ subject in the health management group $(P<.001)$. The disease management program also reduced anxiety symptoms by $4.8 \%$, depression symptoms by $6.6 \%$, current smoking by $14.3 \%$, mortality due to all causes by $9.0 \%$, hospitalizations due to all causes by $16.1 \%$, and emergency department visits due to all causes by $18.1 \%$ $(P<.05$ for all $)$.

Pradella et $\mathrm{al}^{3}$ developed an efficient, low-cost, homebased pulmonary rehabilitation program and evaluated its impact on exercise in 29 subjects with COPD. After subjects performed 24 sessions of exercise (walking for $40 \mathrm{~min}$, climbing stairs for $15 \mathrm{~min}$, and arm exercises for $15 \mathrm{~min}$ ) for $5 \mathrm{~d} /$ week, the authors found that the home-based pulmonary rehabilitation group increased their 6-min walk distance by $65 \mathrm{~m}(P<.05)$ and their endurance test by 316.6 versus $81.8 \mathrm{~m}(P<.05)$ and decreased all St George Respiratory Questionnaire (SGRQ) domains by $>4$ units. In 2014, the Cochrane Collaboration conducted a systematic evaluation of clinical trials evaluating disease management programs and their impact on health outcomes. ${ }^{4}$ Twenty-nine studies that enrolled $>3,000$ subjects were included in the meta-analysis. Self-management programs were associated with a statistically relevant effect on healthrelated quality of life and a lower probability of respiratory-related hospitalization. However, there was no statistically significant effect of self-management on exercise capacity as measured by the 6-min walk test, all-cause hospitalization, or mortality.

\section{Exercise Training}

Exercise training is an essential component of disease management programs because it increases quality of life and exercise tolerance. However, it is important to remember that its positive physiologic effect is a direct correlate of intensity. In most cases, exertional dyspnea prevents patients from sustaining training intensity for the allocated time, making controlled breathing exercises so important. ${ }^{5}$ Ricci et $\mathrm{al}^{6}$ conducted a meta-analysis evaluating noninvasive ventilation as an adjunct during physical training of subjects with COPD who were undergoing pulmonary rehabilitation. Eight studies provided a proper description of a training schedule for subjects with stable COPD. A similar effect between noninvasive ventilation and placebo was observed for the outcomes considered despite differences between studies. However, exercise training was associated with a relevant and statistically significant improvement in almost all of the outcomes, and the improvements in heart rate and work load were significantly correlated to training intensity.

A similar systematic review by Osterling et $\mathrm{al}^{7}$ investigated whether subjects with moderate-to-severe COPD who were participating in pulmonary rehabilitation and exercising at high intensity demonstrated changes in ventilatory parameters associated with decreased dyspnea. Training at high intensity (70-80\% maximum work load) was associated with a statistically significant change in tidal volume and breathing frequency.

\section{Smoking, Depression, and Anxiety in COPD}

In addition to being the most important etiologic factor, smoking is only one of the many issues that affect patients with COPD. Attempts or failures to stop smoking and the effect of COPD on the patient's physical condition, daily activities, and personal relationships make psychiatric disorders common in COPD. Depression and anxiety are known to significantly increase the risk of death in patients with COPD. Lou et $\mathrm{al}^{8}$ evaluated the combined effect of smoking, depression, and anxiety on the mortality of subjects with COPD who participated in a previous study on disease management programs. The combined effect of smoking and depression symptoms increased the death risk by 3.8 -fold. Similarly, the combined effect of smoking and anxiety symptoms increased the death risk by 4.3 -fold.

\section{Respiratory Therapist-Driven Protocols and COPD}

The role of the respiratory therapist (RT) in the management of COPD has been typically limited to the administration of bronchodilator therapy and education on aspects of the disease and pharmacotherapy. With the new changes in reimbursement put in place in 2014 regarding patient readmission, the role of newer management strategies is more important than ever. Whether the implementation of an RT-driven protocol results in better outcomes for patients with COPD has not been properly investigated. Werre et $\mathrm{al}^{9}$ retrospectively compared physiciandirected (non-protocol RT) versus RT-directed respiratory care with regard to hospital stay and 30-d post-discharge 


\section{YEAR IN REVIEW 2014: COPD}

readmission in 244 subjects with COPD who were also diagnosed with acute bacterial pneumonia. Although there were no significant effects on hospital stay between the groups, the frequency of 30-d readmission was significantly lower with RT protocol-directed care $(P=.02)$.

\section{Pharmacotherapy}

Inhaled pharmacotherapy is essential in the management of patients with COPD. The approval of newer and longer-acting agents alone as well as in combination reflects the attempt to optimize therapy for these patients and improve medication adherence. The role of poor inhaler technique cannot be underestimated because even in the best-case scenarios, the more effective medication can simply be wasted. Although a positive response to bronchodilators can help in determining the group of COPD patients with a better clinical outcome, it also indicates a more severe inflammatory process that could compromise exercise endurance. These positive responders have elevated eosinophil levels in induced sputum and higher concentrations of exhaled nitric oxide. Ortega et al ${ }^{10}$ analyzed whether symptoms, quality of life, and exercise capacity varied in 198 subjects with COPD as a function of bronchodilator test results and compared responses to an exercise program. They found that those with positive reversibility had shorter time to exhaustion in the endurance test $(19.1 \pm 12.6 \mathrm{~min}$ vs $24.5 \pm 14.5 \mathrm{~min}, P=.03)$, shorter shuttle walk distance $(380.6 \pm 158.2 \mathrm{~m}$ vs $438.5 \pm 149.1 \mathrm{~m}, P=.02$ ), and lower SGRQ scores $(18.7 \pm 4.6$ vs $19.8 \pm 4.3, P=.01)$. There were no significant differences in peak exercise, peripheral muscle strength, dyspnea, or improvement after exercise training.

The most recent Cochrane meta-analysis ${ }^{11}$ evaluating long-acting inhaled therapy for COPD was published in 2014. The authors randomized 73,062 subjects in 71 trials to over 180 treatment arms, where the long-acting $\beta$-agonist plus inhaled corticosteroid (ICS) combination was ranked the highest. The mean improvement on the SGRQ over placebo was -3.89 units at 6 months and -3.60 at 12 months, and the mean improvement in trough $\mathrm{FEV}_{1}$ over placebo was $133.3 \mathrm{~mL}$ at 6 months $(95 \%$ credible interval [CrI] 100.6-164.0) and slightly less at 12 months (mean difference of 100, 95\% CrI 55.5-140.1). Long-acting muscarinic antagonists were ranked second (SGRQ score of $-2.63,95 \% \mathrm{CrI}-3.53$ to $-1.97 ; \mathrm{FEV}_{1}$ of $103.5 \mathrm{~mL}, 95 \% \mathrm{CrI} 81.8-124.9$ ), and long-acting $\beta$-agonists third (SGRQ score of $-2.29,95 \% \mathrm{CrI}-3.18$ to $-1.53 ; \mathrm{FEV}_{1}$ of $\left.99.4 \mathrm{~mL}, 95 \% \mathrm{CrI} 72.0-127.8\right)$ at 6 months. ICSs were ranked fourth (SGRQ score of -2.00 , $95 \% \mathrm{CrI}-3.06$ to $-0.87 ; \mathrm{FEV}_{1}$ of $65.4 \mathrm{~mL}, 95 \% \mathrm{CrI}$ 33.1-96.9). ${ }^{10}$

Therapy with ICSs has been given consideration as an alternative or adjunct therapy to systemic corticosteroids.
However, a recent evaluation by Crisafulli et al ${ }^{12}$ demonstrated a lack of ICS influence in the early systemic inflammatory response to and clinical presentation of 123 hospitalized subjects with COPD exacerbations. However, ICS withdrawal was recently found to be associated with a greater decrease in lung function. In a recent 12-month, double-blind, parallel-group study, Magnussen et al ${ }^{13}$ evaluated 2,485 subjects with a history of COPD exacerbations who received triple therapy (tiotropium, salmeterol, and fluticasone propionate) during a 6-week run-in period. Subjects were then randomly assigned to continued triple therapy or withdrawal of ICS over a 12-week period. When ICS withdrawal was complete, the mean reduction from baseline in the trough $\mathrm{FEV}_{1}$ was $38 \mathrm{~mL}$ greater in the ICS-withdrawal group than in the ICS-continuation group $(P<.001)$. This between-group difference was also observed at week 52. The controversial issue of the significant reduction of COPD exacerbations but also the higher risk of pneumonia associated with the addition of ICSs to long-acting bronchodilator therapy should always be considered. ${ }^{14}$

Regardless of the inhaled medication selected for the management of COPD, improving inhaler technique and adherence should be key targets for health-care professionals working with these patients. As recommended by van Boven et al ${ }^{15}$ in their cost-effectiveness analysis, close monitoring of inhalation technique and medication adherence should be considered before adding new inhaled medications to a therapeutic regimen that is already cumbersome for some patients with COPD. However, demonstration of proper inhaler technique by health-care providers may be as important as patients' ability to use these devices. ${ }^{16}$ Basheti et al ${ }^{17}$ assessed the ability of 200 healthcare professionals, including 10 pulmonologists, 46 general practitioners, 79 pharmacists, 15 pharmacist assistants, 40 nurses, and 10 RTs, in Jordan to demonstrate the correct use of some commonly used inhalers following standardized checklists. Pulmonologists scored highest on baseline inhaler technique demonstration skills $(8.5 / 10)$, whereas the other health-care professionals scored poorly in demonstrating the correct use of a dry powder inhaler compared with a pressurized metered-dose inhaler. RTs scored 2.83 on both dry powder inhalers (Diskus and Turbuhaler) and pressurized metered-dose inhalers, only slightly better than pharmacists. Although training of this group resulted in significant improvement in inhaler technique by a mean of almost 4 points, the RTs continued to score below every other group. ${ }^{17}$

\section{Summary}

The management of patients with COPD has increased in complexity due to a larger number of available medications. A significant number of publications in 2014 con- 


\section{YEAR IN REVIEW 2014: COPD}

firmed the importance of disease management programs and exercise training in improving health-related outcomes and quality of life. Every attempt should be made to ensure that patients with COPD obtain adequate psychiatric evaluation and management of depression and anxiety because this can make a difference between life and death. Finally, inhaled medications are only as good as the inhaler technique. A qualified health-care professional should be able to demonstrate competence in the use of the most common inhalers. Instructing patients on the right technique is not only cost-effective but also improves medication adherence and significantly changes clinical outcomes.

\section{REFERENCES}

1. Mehring M, Donnachie E, Fexer J, Hofmann F, Schneider A. Disease management programs for patients with COPD in Germany: a longitudinal evaluation of routinely collected patient records. Respir Care 2014;59(7):1123-1132.

2. Lou P, Chen P, Zhang P, Yu J, Wang Y, Chen N, et al. A COPD health management program in a community-based primary care setting: a randomized controlled trial. Respir Care 2015;60(1):102-112.

3. Pradella CO, Belmonte GM, Maia MN, Delgado CS, Luise AP, Nascimento OA, et al. Home-based pulmonary rehabilitation for subjects with COPD: a randomized study. Respir Care 2015;60(4): 526-532.

4. Zwerink M, Brusse-Keizer M, van der Valk PD, Zielhuis GA, Monninkhof EM, van der Palen J, et al. Self management for patients with chronic obstructive pulmonary disease. Cochrane Database Syst Rev. 2014;3:CD002990.

5. Borge CR, Hagen KB, Mengshoel AM, Omenaas E, Moum T, Wahl AK. Effects of controlled breathing exercises and respiratory muscle training in people with chronic obstructive pulmonary disease: results from evaluating the quality of evidence in systematic reviews. BMC Pulm Med 2014;14:184.

6. Ricci C, Terzoni S, Gaeta M, Sorgente A, Destrebecq A, Gigliotti F. Physical training and noninvasive ventilation in COPD patients: a meta-analysis. Respir Care 2014;59(5):709-717.
7. Osterling K, MacFadyen K, Gilbert R, Dechman G. The effects of high intensity exercise during pulmonary rehabilitation on ventilatory parameters in people with moderate to severe stable COPD: a systematic review. Int J Chron Obstruct Pulmon Dis 2014;9:10691078.

8. Lou P, Chen P, Zhang P, Yu J, Wang Y, Chen N, et al. Effects of smoking, depression, and anxiety on mortality in COPD patients: a prospective study. Respir Care 2014;59(1):54-61.

9. Werre ND, Boucher EL, Beachey WD. Comparison of therapistdirected and physician-directed respiratory care in COPD subjects with acute pneumonia. Respir Care 2015;60(2):151-154.

10. Ortega F, Márquez-Martín E, Valencia B, Cejudo P, Rodriguez A, López-Campos JL, Barrot E. Impact of bronchodilator responsiveness on quality of life and exercise capacity in patients with COPD. Respir Care 2014;59(1):81-89.

11. Kew KM, Dias S, Cates CJ. Long-acting inhaled therapy (betaagonists, anticholinergics and steroids) for COPD: a network metaanalysis. Cochrane Database Syst Rev 2014;3:CD010844.

12. Crisafulli E, Guerrero M, Menéndez R, Huerta A, Martinez R, Gimeno A, et al. Inhaled corticosteroids do not influence the early inflammatory response and clinical presentation of hospitalized subjects with COPD exacerbation. Respir Care 2014;59(10):1550-1559.

13. Magnussen H, Disse B, Rodriguez-Roisin R, Kirsten A, Watz H, Tetzlaff K, et al. Withdrawal of inhaled glucocorticoids and exacerbations of COPD. N Engl J Med. 2014;371(14):1285-1294

14. DiSantostefano RL, Li H, Hinds D, Galkin DV, Rubin DB. Risk of pneumonia with inhaled corticosteroid/long-acting $\beta 2$ agonist therapy in chronic obstructive pulmonary disease: a cluster analysis. Int J Chron Obstruct Pulmon Dis 2014;9:457-468.

15. van Boven JF, Tommelein E, Boussery K, Mehuys E, Vegter S, Brusselle GG, et al. Improving inhaler adherence in patients with chronic obstructive pulmonary disease: a cost-effectiveness analysis. Respir Res 2014;15:66.

16. Basheti IA, Bosnic-Anticevich SZ, Armour CL, Reddel HK. Checklists for powder inhaler technique: a review and recommendations. Respir Care 2014;59(7):1140-1154.

17. Basheti IA, Qunaibi EA, Hamadi SA, Reddel HK. Inhaler technique training and health-care professionals: effective long-term solution for a current problem. Respir Care 2014;59(11):1716-1725. 